\section{Gravidez na adolescência e características socioeconômicas dos municípios do Estado de São Paulo, Brasil: análise espacial}

\author{
Teenage pregnancy rates and socioeconomic \\ characteristics of municipalities in São Paulo State, \\ Southeast Brazil: a spatial analysis
}

1. Faculdade de Medicina de
Ribeirão Preto, Universidade
de São Paulo, São Paulo,
Brasil.
Correspondência
E. Z. Martinez
Departamento de Medicina
Social, Faculdade de
Medicina de Ribeirão Preto,
Universidade de São Paulo.
Av. Bandeirantes 3900,
Ribeirão Preto, SP 14049-900,
Brasil.
edson@fmrp.usp.br

Abstract

Teenage pregnancy is a common public health problem worldwide. The objective of this ecological study was to investigate the spatial association between teenage pregnancy rates and socioeconomic characteristics of municipalities in São Paulo State, Southeast Brazil. We used a Bayesian model with a spatial distribution following $a$ conditional autoregressive (CAR) form based on Markov Chain Monte Carlo algorithm. We used data from the Live Birth Information System (SINASC) and the Brazilian Institute of Geography and Statistics (IBGE). Early pregnancy was more frequent in municipalities with lower per capital gross domestic product (GDP), higher poverty rate, smaller population, lower human development index (HDI), and a higher percentage of individuals with State social vulnerability index of 5 or 6 (more vulnerable). The study demonstrates a significant association between teenage pregnancy and socioeconomic indicators.

Pregnancy in Adolescence; Health Status Indicators; Spatial Analysis
Edson Zangiacomi Martinez ${ }^{1}$

Daiane Leite da Roza 1

Maria do Carmo Gullaci Guimarães

Caccia-Bava ${ }^{1}$

Jorge Alberto Achcar 1

Amaury Lelis Dal-Fabbro 1

\section{Introdução}

Dentro do processo de crescimento e desenvolvimento humano, a adolescência é uma fase marcada por intensas transformações físicas e biológicas, que se associam a outras de âmbito social, emocional, cultural e psicológico, de forma que o corpo assume uma dimensão bastante significava na vida da adolescente ${ }^{1}$. Até a transição entre a infância e a adolescência, as relações afetivas de maior importância tendem a ser as familiares. No entanto, essas relações modificam-se quando as características sexuais secundárias começam a surgir como resultado da estimulação hormonal 2, o que favorece uma etapa marcada por namoros e o início de experiências sexuais. Consequentemente, as adolescentes são expostas ao risco de gravidezes precoces, sendo a magnitude deste risco associada a fatores sociais e econômicos 3 .

Enquanto, durante muito tempo, a adolescência foi considerada a etapa ideal para engravidar, hoje é considerada uma idade inadequada para a mulher ter filhos ${ }^{4}$, perante as associações da gravidez precoce com morbidades do neonato e impactos econômicos, educacionais e sociais 5. Como exemplo, uma consequência da gravidez precoce bastante explorada na literatura é o baixo peso ao nascer 6,7. Essa associação tem por mecanismos fatores como a imaturidade do sistema reprodutivo e o ganho 
de peso inadequado durante a gestação ${ }^{6}$, além de aspectos como a pobreza, falta de instrução e cuidados pré-natais. Outro fator decorrente da gravidez na adolescência é a prematuridade (idade gestacional abaixo de 37 semanas) ${ }^{8}$, que pode predispor o recém-nascido a infecções ou a problemas como hipoglicemia, hipóxia e atraso no desenvolvimento neuropsicomotor futuro 9 .

A gestação na adolescência tem sido considerada um importante assunto de saúde pública, em virtude da sua alta prevalência 3 . Segundo a Pesquisa Nacional sobre Demografia e Saúde (PNDS) 10, realizada em 1996, 18\% das adolescentes brasileiras de 15 a 19 anos já tiveram pelo menos um filho ou estavam grávidas. Nos estudos dos fatores associados às causas e consequências dessa gravidez é necessário considerar que se trata de um fenômeno complexo, associado a fatores econômicos, educacionais e comportamentais 11. A literatura tem evidenciado associações entre esse fenômeno e variáveis, como a desigualdade social e econômica 6,12,13, início precoce da vida sexual 14, história materna de gravidez na adolescência 14 , pré-natal inadequado 13, não utilização ou utilização inconsistente de métodos contraceptivos 15,16 e uso frequente de drogas ilícitas por familiares 17.

Considerando-se que a gravidez, assim como outros fenômenos sociais, difere por regiões e grupos sociais, é assumida a hipótese de não aleatoriedade espacial da gravidez adolescente em diferentes ambientes 18. A despeito da importância do tema, a literatura evidencia uma lacuna em pesquisas que considerem o aspecto espacial da distribuição desse fenômeno. Assim, o presente trabalho tem por objetivo estudar a associação entre os percentuais de gravidez na adolescência e características socioeconômicas e de vulnerabilidade social dos municípios do Estado de São Paulo, Brasil, considerando uma estrutura espacial para os dados.

\section{Métodos}

Neste estudo ecológico, avaliou-se a associação entre os percentuais de gravidez na adolescência e as seguintes características de cada um dos 645 municípios do Estado de São Paulo: número médio de anos de estudo dos responsáveis pelos domicílios (segundo Censo Demográfico de 2000. Instituto Brasileiro de Geografia e Estatística - IBGE; http://www.ibge.gov.br), tamanho da população (em número de habitantes, estimado pelo IBGE para 2007), produto interno bruto (PIB) per capita (dados de 2007, em Reais), índice de Gini 19 (medida do grau de concentração de renda, cujos valores variam de 0 a 1 , sendo este último correspondente à desigualdade máxima), incidência de pobreza (segundo mapa de pobreza e desigualdade do IBGE, calculada para o ano de 2003), índice municipal de desenvolvimento humano (IDH-M) e índice paulista de vulnerabilidade social (IPVS) 20.

Os dados de gravidezes na adolescência foram obtidos no Sistema de Informações sobre Nascidos Vivos (SINASC) do Ministério da Saúde, considerando o número total de nascidos vivos em cada município do Estado de São Paulo no ano de 2007 e o número de nascidos vivos cuja mãe tinha até 19 anos, nos respectivos municípios 21 . A média de anos de estudos dos responsáveis pelos domicílios, o tamanho da população (em número de habitantes), o PIB per capita, o índice de Gini e a incidência de pobreza foram obtidos na página eletrônica do IBGE (http:// www.ibge.gov.br).

O IPVS foi criado pela Fundação Sistema Estadual de Análise de Dados (Fundação Seade) para identificar os segmentos populacionais mais vulneráveis à pobreza, nos 645 municípios do Estado de São Paulo 22. De acordo com os setores censitários, o IPVS classifica os segmentos populacionais de um município em seis estratos: nenhuma vulnerabilidade (grupo 1), vulnerabilidade muito baixa (grupo 2), baixa (grupo 3), média (grupo 4), alta (grupo 5) e muito alta (grupo 6). No presente estudo, utilizou-se como indicador de vulnerabilidade social o percentual de indivíduos de cada município classificados nos grupos 5 e 6 do IPVS, por englobarem os setores censitários que possuem as piores condições na dimensão socioeconômica (baixa), nos quais os chefes de domicílios apresentam, em média, os níveis mais baixos de renda e escolaridade 23 .

O IDH-M é derivado do IDH utilizado pelo Programa das Nações Unidas para o Desenvolvimento (PNUD). Esse índice é composto por indicadores capazes de influenciar a qualidade de vida dos moradores de um município, sendo calculado pela média aritmética de três componentes: educação, longevidade e renda. O componente educação considera o índice de analfabetismo do município e a taxa de matrícula em todos os níveis de ensino. O componente longevidade é baseado na expectativa de vida ao nascer, enquanto o componente renda é dado pelo PIB do município, corrigido pelo poder de compra da moeda local. Uma lista contendo o IDH-M para os municípios brasileiros, relativo ao ano 2000, está disponível na página eletrônica do PNUD (http://www.pnud.org.br/atlas/ tabelas/index.php). O IDH-M e seus componentes são padronizados em uma escala de 0 a 1 , sendo que maiores valores são indicativos de maior desenvolvimento. 
A análise estatística dos dados considerou que, para o município $i, Y_{i}$ é a contagem de nascimentos de mães adolescentes, $N_{i}$ é o número total de nascidos vivos e $\theta_{i}$ é o percentual de gravidezes na adolescência. Assim, o modelo estatístico considerou que $Y_{i}$ é uma variável aleatória que segue uma distribuição binomial com probabilidade $\theta_{i}$ em $N_{i}$ ensaios independentes. Formalmente, o modelo estatístico foi escrito na forma:

$Y_{i} \mid N_{i}, \theta_{\mathrm{i}} \sim \operatorname{Binomial}\left(N_{i}, \theta_{i}\right), i=1, \ldots, M$,

em que, $M=645$ é o número de municípios, $N_{i}$ foi considerado conhecido e $\theta_{i}$ é um parâmetro a ser estimado pelo modelo. Foi assumida uma função de ligação logito entre os percentuais de gravidez na adolescência $\theta_{i}$ e uma observação $x_{i}$ da variável quantitativa $X$, escrita na forma:

$\theta_{i=} \frac{\exp \left[\alpha+\beta\left(x_{i}-\bar{x}\right)+\gamma_{i}\right]}{1+\exp \left[\alpha+\beta\left(x_{i}-\bar{x}\right)+\gamma_{i}\right]}$,

em que, $\bar{x}$ é a média aritmética das observações $x_{1}, x_{2}, \ldots, x_{645}, a$ e $b$ são parâmetros desconhecidos (efeitos fixos) e $\gamma_{i}$ é um efeito aleatório associado ao $i$-ésimo município. $\mathrm{Na}$ análise bayesiana 24 , considerou-se que $\gamma_{i}$ assume uma distribuição $a$ priori espacial que permite que as correlações entre as áreas próximas no espaço sejam maiores. A distribuição espacial aqui adotada seguiu uma estrutura condicional autorregressiva (CAR) 25,26 tal que:

$\gamma_{i} \mid\left\{\gamma_{j}, i \neq j, j \in A^{*}(i)\right\}, \sigma_{\gamma}^{2} \sim N\left(\bar{\eta}_{i}, \frac{\sigma_{\gamma}^{2}}{n_{i}}\right)$

em que, $A^{*}(i)$ denota o conjunto dos municípios vizinhos ao município $i, \bar{\eta}_{i}$ é a média dos efeitos aleatórios $\gamma_{j}$ associados aos municípios vizinhos ao município $i, n_{i}$ corresponde ao número de municípios que são vizinhos ao município $i, \sigma_{\gamma}^{2}$ é a variância da distribuição e $N(a, b)$ genericamente denota uma distribuição normal com média $a$ e variância $b$. O critério utilizado para a definição de vizinhança foi o de adjacência, no qual áreas que fazem fronteira umas com as outras foram consideradas vizinhas. O Município de Ilhabela, embora situado integralmente em uma ilha, foi considerado adjacente ao Município de São Sebastião por sua proximidade.

Considerando a forte presença de colinearidade entre as variáveis socioeconômicas e de vulnerabilidade social, não foram ajustados modelos múltiplos, mas um modelo para cada variável. Para o ajuste do modelo, utilizou-se o módulo GeoBUGS 27 do programa computacional WinBUGS 28 (Medical Research Council, Biostatistics Unit, Cambridge, Reino Unido). Assumiuse que o efeito fixo $a$ segue uma distribuição $a$ priori do tipo flat imprópria, definida sobre todo o conjunto dos números reais, e o efeito fixo $b$ segue uma distribuição normal não informativa, com média igual a 0 e variância igual a 1.000 . O uso da distribuição normal para a especificação dessas distribuições a priori é justificado por diversos autores 29,30. E ainda, assumiu-se que a variância $\sigma_{\gamma}^{2}$ segue $a$ priori uma distribuição gama inversa, expressa por $\sigma_{\gamma}^{2} \sim \operatorname{Gama}(0,5 ; 0,0005)$, conforme recomendação da literatura 27 . Considerou-se que essas distribuições a priori são, entre si, independentes. Dado que, nesse modelo, as distribuições condicionais a posteriori possuem alguma complexidade em suas formas, métodos computacionalmente intensivos foram utilizados para a simulação de amostras dessas distribuições. Mais especificamente, utilizou-se o algoritmo Monte Carlo em cadeias de Markov (MCMC) para a obtenção de amostras dessas distribuições. Foram geradas 605 mil amostras de cada parâmetro de interesse, sendo descartadas as 5 mil primeiras para evitar algum efeito dos valores iniciais (burn-in samples). Para prevenir correlações entre amostras sucessivamente geradas, utilizou-se para as inferências saltos tamanho 150.

Para verificar se modelos que incluem os efeitos aleatórios $\gamma_{j}$ para descrever a estrutura espacial apresentam melhor desempenho que modelos similares, sem os efeitos $\gamma_{j}$, utilizou-se o critério DIC (deviance information criterion) ${ }^{31}$. Esse critério estabelece que modelos que apresentam menores valores de DIC são aqueles com melhor ajuste aos dados.

\section{Resultados}

Para o ano de 2007, o percentual de gravidez na adolescência no Estado de São Paulo foi de $16,4 \%$. A Figura 1 apresenta os percentuais brutos de gravidez na adolescência segundo os 645 municípios do estado. As menores porcentagens brutas de gravidez na adolescência corresponderam aos municípios de Santa Rosa de Viterbo, Uru, Elisário, Miracatu, Muritinga do Sul, Águas de São Pedro, Mariápolis, Floreal, Sagres, São Caetano do Sul e Santa Rita do Passa Quatro. Em todos esses municípios, os percentuais foram menores que $10 \%$. As maiores taxas brutas foram identificadas nos municípios de Pedranópolis, João Ramalho, Cássia dos Coqueiros, Ubarana, São João das Duas Pontes e Pracinha, todos com percentuais superiores a 40\%. Dentre os municípios paulistas com população superior a $100 \mathrm{mil}$ habitantes, as menores porcentagens de gravidez na adolescência corresponderam a São Caetano do Sul, Ribeirão Pires, Americana, Santos, Santo André, Jundiaí, Ribeirão Preto, São Bernardo 


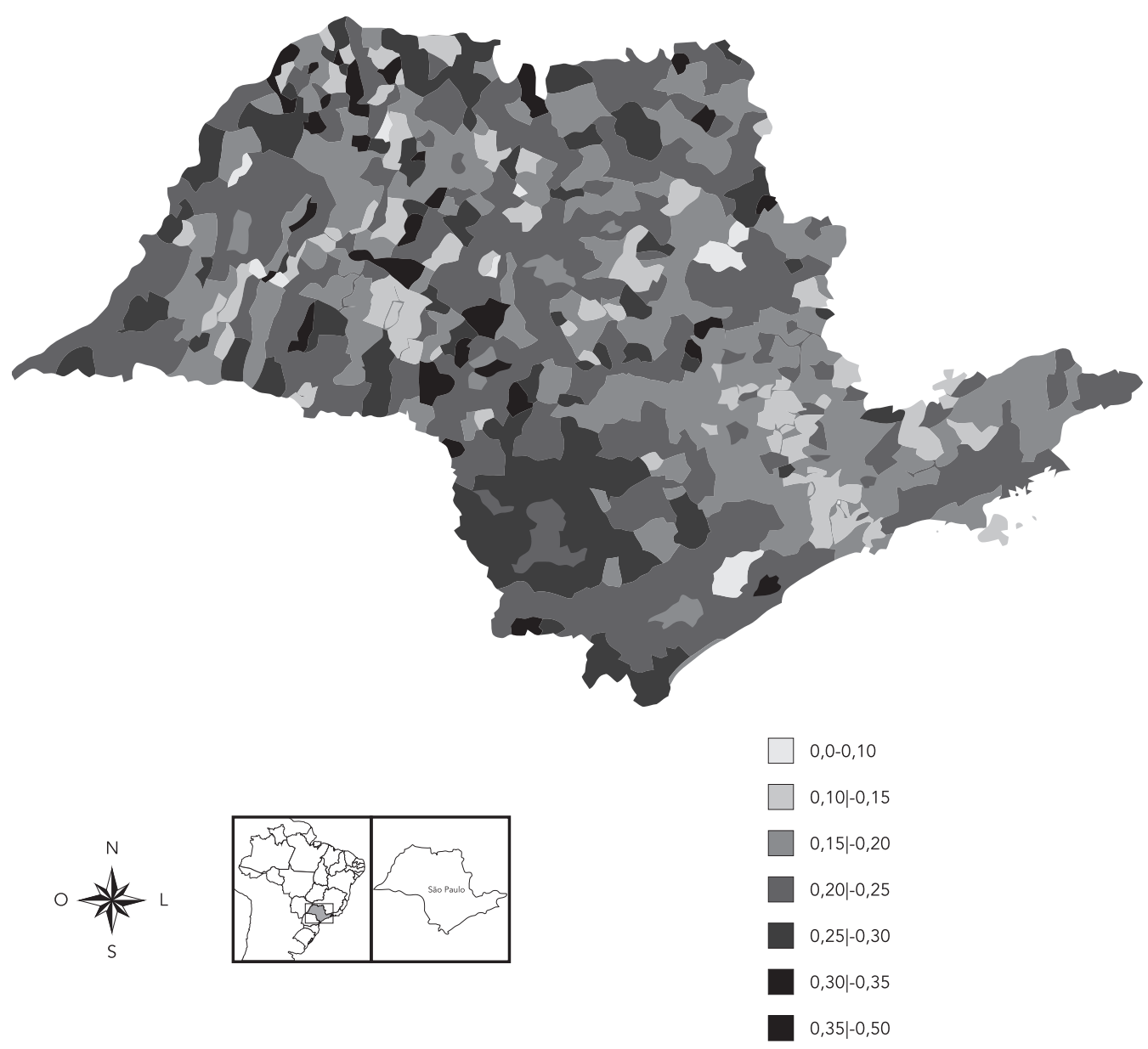

do Campo, Santa Bárbara d'Oeste e Campinas; e as maiores porcentagens a Itapecerica da Serra, Araras, Cubatão, Itapevi, Tatuí, Guarujá, Barretos, Itapetininga, Itaquaquecetuba e Francisco Morato.

Os valores esperados a posteriori dos percentuais de gravidez na adolescência, ajustados pelo modelo bayesiano, podem ser vistos na Figura 2. Enquanto a Figura 1 traz uma aparência de "colcha de retalhos", não evidenciando de forma clara um padrão espacial para a distribuição dos percentuais de gravidez na adolescência, a visualização da Figura 2 sugere uma suavização dos percentuais, evidenciando que os municípios de maior porte (como Ribeirão Preto, Araraquara, São José dos Campos, Taubaté, Presidente Prudente, Campinas e a região metropolitana de São
Paulo) tendem a possuir menores percentuais ajustados de gravidez na adolescência, enquanto a localização dos municípios com maiores percentuais tende a ser afastada das cidades mais populosas.

A Figura 3 descreve a dispersão dos percentuais de gravidez na adolescência e: (a) incidência de pobreza; (b) média de anos de estudos dos responsáveis pelos domicílios; (c) índice de Gini; (d) PIB; (e) tamanho da população; e (f) percentual de indivíduos com IPVS igual a 5 ou 6 . As curvas sobrepostas aos gráficos foram obtidas por modelos de regressão descritos na seção anterior, considerando uma estrutura condicional autorregressiva para os efeitos aleatórios.

A Tabela 1 descreve os percentuais médios de gravidez na adolescência segundo classes das 
Distribuição espacial dos percentuais ajustados de gravidez na adolescência nos municípios do Estado de São Paulo, Brasil, obtidos pelo modelo bayesiano.

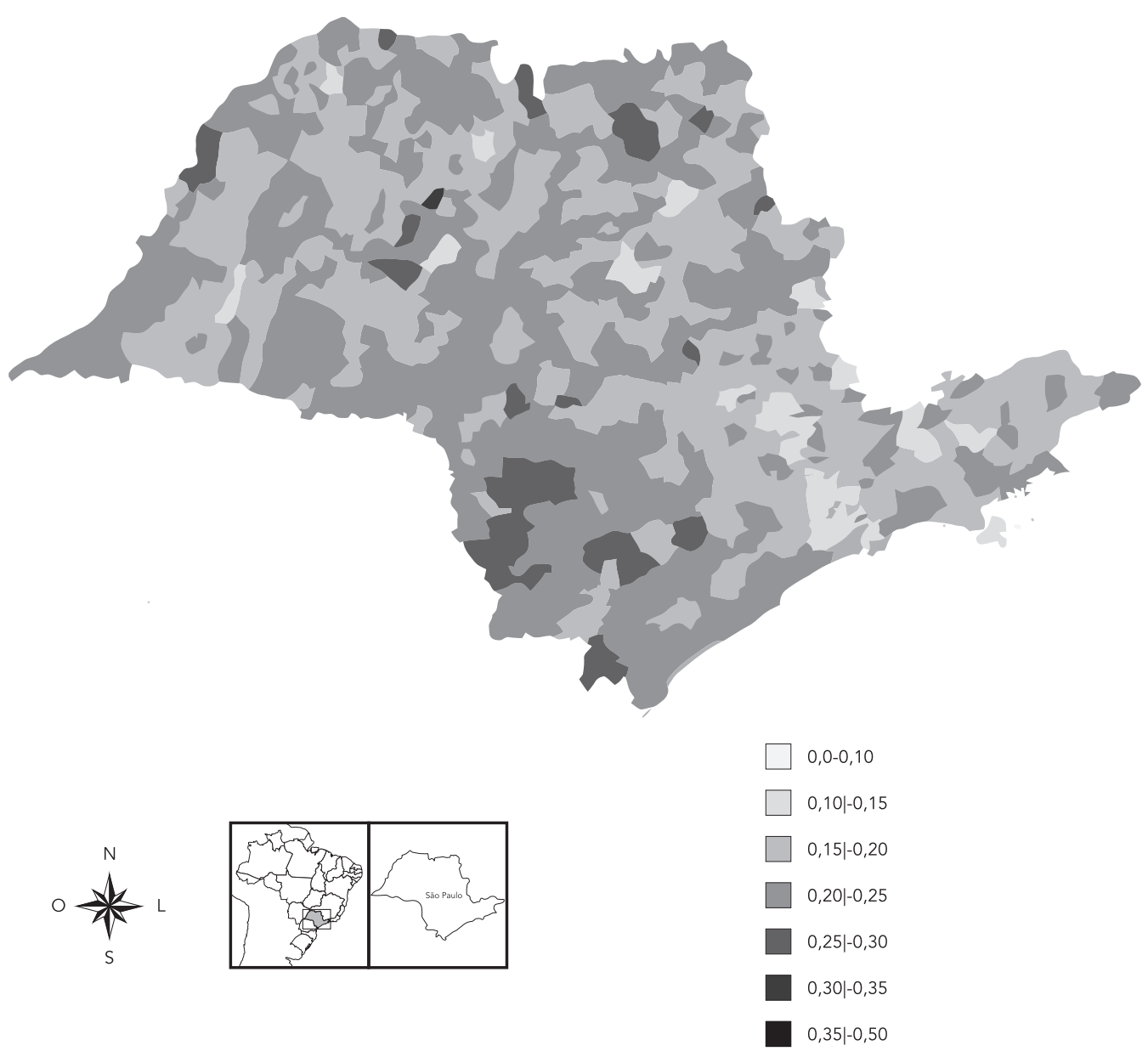

variáveis socioeconômicas que caracterizam o Estado de São Paulo. Cada variável foi classificada em seis classes. Na primeira, encontra-se, aproximadamente, o equivalente a $5 \%$ dos municípios com os menores valores de cada variável. A segunda classe engloba os municípios relativos aos valores situados entre os percentis 5\% e $25 \%$, a terceira abrange os municípios com os valores situados entre os percentis $25 \%$ e $50 \%$, a quarta os situados entre os percentis $50 \%$ e $75 \%$ e a quinta classe os municípios relativos aos valores situados entre os percentis $75 \%$ e $95 \%$. A última classe equivale aproximadamente aos 5\% dos municípios com os maiores valores de cada variável. Essa classificação permite, dessa forma, comparações entre os extremos de cada variável.
O índice de Gini e os escores do IPVS foram descritos em cinco níveis, devido à distribuição dos valores destas variáveis.

Os percentuais médios de gravidez na adolescência exibidos na Tabela 1 foram encontrados por modelos de regressão como descritos na seção anterior, em que a variável independente foi substituída por variáveis indicadoras (dummies variables), permitindo assim estimar os percentuais para cada classe da variável e obter as razões entre os percentuais, com os seus respectivos intervalos de $95 \%$ de credibilidade. Intervalos de credibilidade que não incluem o valor 1 indicam associações significativas com os percentuais de gravidez na adolescência (indicados com asteriscos na Tabela 1, o que é 
Figura 3

Diagramas de dispersão dos percentuais de gravidez na adolescência nos municípios do Estado de São Paulo, Brasil, e (a) incidência de pobreza; (b) média de anos de estudos dos responsáveis pelos domicílios; (c) índice de Gini; (d) PIB per capita; (e) tamanho da população; ( $f$ ) percentual de indivíduos com IPVS igual a 5 ou 6 .

a) Incidência de pobreza

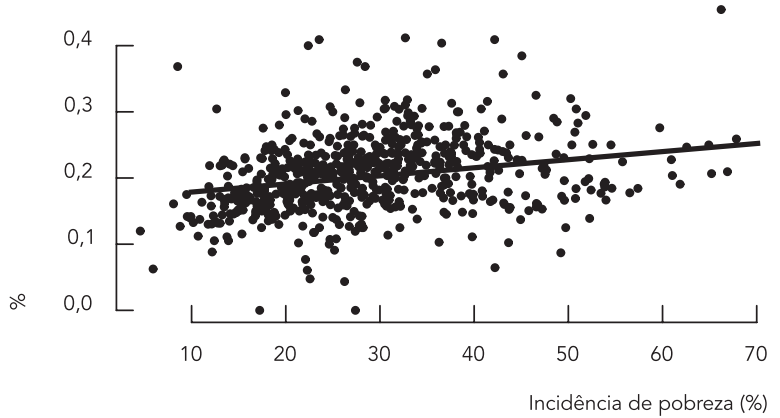

c) Índice de Gini

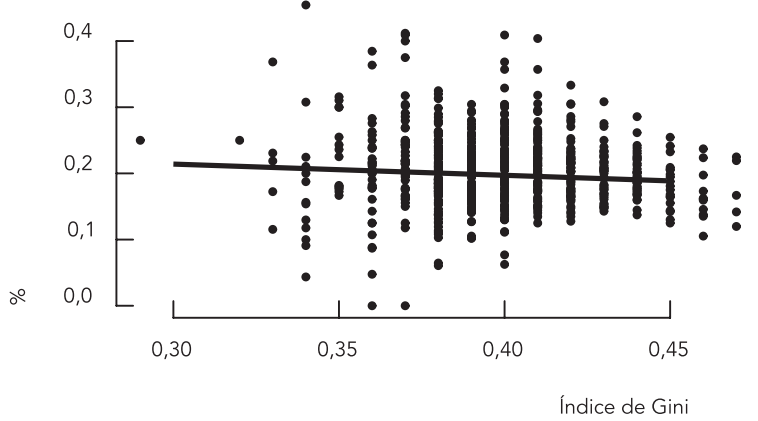

e) Tamanho da população

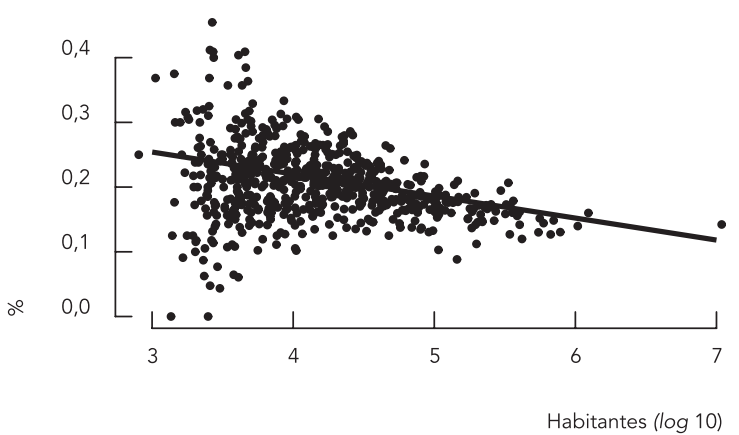

b) Média de anos de estudos dos responsáveis pelos domicílios

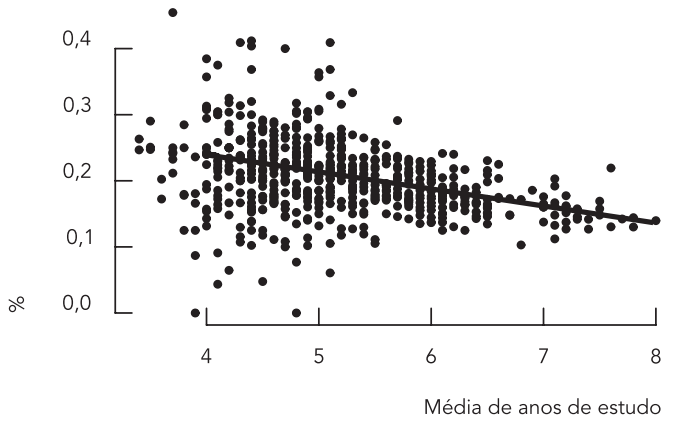

d) PIB per capita

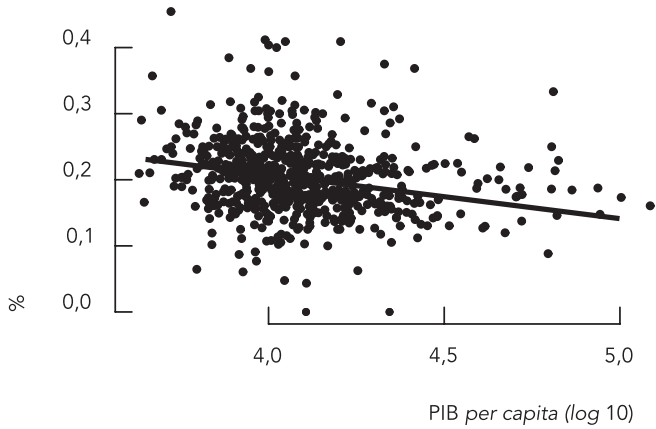

f) Percentual de indivíduos com IPVS iguais a 5 ou 6

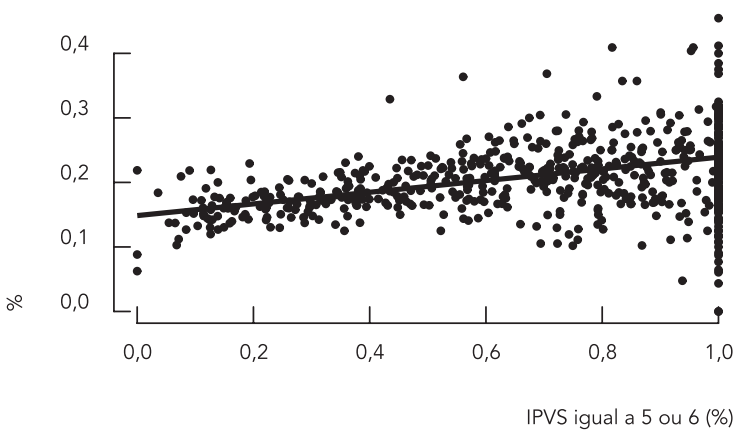

IPVS: índice paulista de vulnerabilidade social; PIB: produto interno bruto.

Nota: as curvas exibidas nos gráficos são resultantes do ajuste dos modelos de regressão. 
Percentuais médios de gravidez na adolescência, segundo características dos municípios do Estado de São Paulo, Brasil.

\begin{tabular}{|c|c|c|c|c|c|}
\hline \multirow[t]{2}{*}{ Variáveis } & \multirow[t]{2}{*}{$\begin{array}{l}\text { Número de } \\
\text { municípios }\end{array}$} & \multicolumn{2}{|c|}{$\begin{array}{l}\text { Percentual médio de } \\
\text { gravidez na adolescência }\end{array}$} & \multicolumn{2}{|c|}{ Razão entre os percentuais } \\
\hline & & $\%$ & IC95\% & Razão & IC95\% \\
\hline \multicolumn{6}{|c|}{ Escolaridade média do responsável } \\
\hline \multicolumn{6}{|c|}{ pelo domicílio (anos de estudos) } \\
\hline Até 4 & 35 & 21,16 & $19,72-22,72$ & \multicolumn{2}{|c|}{ Referência } \\
\hline $4,1-4,5$ & 119 & 22,70 & $21,68-23,70$ & 1,074 & $0,989-1,160$ \\
\hline $4,6-5,0$ & 158 & 22,26 & $21,59-22,96$ & 1,054 & $0,973-1,138$ \\
\hline $5,1-5,8$ & 184 & 20,31 & $19,84-20,81$ & 0,961 & $0,887-1,036$ \\
\hline $5,9-7,0$ & 114 & 17,35 & $16,92-17,79$ & 0,821 & $0,759-0,888$ * \\
\hline$>7,0$ & 35 & 14,56 & $13,94-15,18$ & 0,689 & $0,636-0,750$ * \\
\hline \multicolumn{6}{|c|}{ Incidência da pobreza (\%) } \\
\hline Até 13,45 & 33 & 14,38 & $13,44-15,30$ & \multicolumn{2}{|c|}{ Referência } \\
\hline $13,46-20,43$ & 129 & 17,67 & $17,02-18,20$ & 1,230 & $1,140-1,346$ * \\
\hline $20,44-27,01$ & 161 & 19,79 & $19,28-20,30$ & 1,378 & $1,290-1,493$ * \\
\hline $27,02-34,72$ & 161 & 21,92 & $21,27-22,59$ & 1,527 & $1,430-1,655$ * \\
\hline $34,73-50,37$ & 129 & 20,53 & $19,73-21,47$ & 1,430 & $1,323-1,551$ * \\
\hline$>50,37$ & 32 & 20,55 & $19,36-21,96$ & 1,431 & $1,305-1,561$ * \\
\hline \multicolumn{6}{|l|}{ Índice de Gini } \\
\hline Até 0,35 & 34 & 20,20 & $18,17-22,16$ & \multicolumn{2}{|c|}{ Referência } \\
\hline $0,36-0,38$ & 173 & 19,65 & $18,88-20,45$ & 0,975 & $0,879-1,091$ \\
\hline $0,39-0,40$ & 173 & 19,63 & $19,00-20,28$ & 0,975 & $0,878-1,086$ \\
\hline $0,41-0,44$ & 230 & 20,05 & $20,05-20,54$ & 0,995 & $0,900-1,109$ \\
\hline$>0,44$ & 35 & 16,89 & $16,89-17,92$ & 0,839 & $0,752-0,940$ * \\
\hline \multicolumn{6}{|l|}{ PIB per capita } \\
\hline Até 6.444 & 33 & 22,72 & $21,12-24,47$ & \multicolumn{2}{|c|}{ Referência } \\
\hline $6.445-8.940$ & 129 & 21,30 & $20,49-22,14$ & 0,939 & $0,862-1,018$ \\
\hline $8.941-11.711$ & 161 & 20,92 & $20,26-21,59$ & 0,922 & $0,847-0,998$ * \\
\hline $11.712-16.122$ & 161 & 19,48 & $18,90-20,07$ & 0,858 & $0,790-0,928$ * \\
\hline $16.123-34.501$ & 129 & 17,39 & $16,82-17,97$ & 0,766 & $0,704-0,829$ * \\
\hline$>34.501$ & 32 & 16,49 & $15,43-17,60$ & 0,727 & $0,655-0,801$ * \\
\hline \multicolumn{6}{|c|}{ Tamanho da população (habitantes) } \\
\hline Até 2.231 & 33 & 21,89 & $18,95-24,82$ & \multicolumn{2}{|c|}{ Referência } \\
\hline $2.232-4.881$ & 129 & 21,53 & $20,44-22,58$ & 0,988 & $0,858-1,137$ \\
\hline $4.882-12.226$ & 161 & 21,53 & $20,79-22,30$ & 0,988 & $0,862-1,136$ \\
\hline $12.227-35.903$ & 161 & 21,20 & $20,64-21,77$ & 0,973 & $0,847-1,117$ \\
\hline $35.904-207.028$ & 129 & 17,67 & $17,25-18,08$ & 0,811 & $0,709-0,930$ * \\
\hline$>207.028$ & 32 & 15,22 & $14,44-15,98$ & 0,699 & $0,601-0,812$ * \\
\hline \multicolumn{6}{|c|}{ IPVS, escores iguais a 5 ou 6 (\%) } \\
\hline Até 14,9 & 35 & 14,61 & $13,98-15,24$ & \multicolumn{2}{|c|}{ Referência } \\
\hline $15,0-45,6$ & 126 & 17,43 & $16,96-17,91$ & 1,194 & $1,133-1,259$ * \\
\hline $45,7-74,3$ & 161 & 20,64 & $20,15-21,14$ & 1,414 & $1,343-1,489$ * \\
\hline $74,4-99,9$ & 163 & 22,08 & $21,46-22,72$ & 1,513 & $1,435-1,593$ * \\
\hline 100,0 & 160 & 21,72 & $20,78-22,67$ & 1,488 & $1,396-1,584$ * \\
\hline
\end{tabular}

IC95\%: intervalo de 95\% de credibilidade; IPVS: índice paulista de vulnerabilidade social; PIB: produto interno bruto.

* Diferenças significantes em relação à classe de referência. 
análogo a estabelecer um nível de significância de $5 \%$ quando utilizados métodos frequentistas de inferência estatística). Com exceção do índice de Gini, em um contexto espacial todas as variáveis socioeconômicas e de vulnerabilidade social aqui estudadas associaram-se aos percentuais de gravidez na adolescência.

A Figura 4 compõe-se de quatro painéis, que exibem gráficos de dispersão dos percentuais de gravidez na adolescência, e o IDH-M e seus componentes no painel (a). No painel (b), apresentase o componente educação; no (c), o componente longevidade; e no painel (d), o componente renda. As curvas sobrepostas aos gráficos foram obtidas dos modelos de regressão descritos na seção anterior, evidenciando significativos decréscimos dos percentuais de gravidez precoce, conforme é observado um aumento dos índices de desenvolvimento dos municípios. Por sua vez, a Tabela 2 descreve os percentuais médios de gravidez na adolescência segundo classes do IDH-M e de seus componentes, sendo estes índices classificados em seis faixas, de maneira análoga às categorizações usadas na Tabela 1. A Tabela 2 descreve uma progressiva redução dos percentuais de gravidez precoce conforme aumentam os valores do IDH.

Em todas as análises verificou-se que os modelos que utilizaram a estrutura espacial apresentaram valores menores para o DIC do que os modelos que desprezam tal estrutura. Esse resultado evidencia que os modelos que utilizaram a estrutura espacial são mais adequados aos dados do presente estudo, ou seja, não há uma aleatoriedade espacial dos percentuais de gravidez na adolescência entre os diferentes municípios.

\section{Discussão}

O presente estudo aponta a estreita relação entre a gravidez na adolescência e fatores econômicos e sociais no Estado de São Paulo. Os percentuais de gravidez na adolescência apresentaram-se maiores nos municípios de menor PIB, maior incidência de pobreza, menor tamanho populacional e maior percentual de indivíduos com IPVS igual a 5 ou 6, ou seja, mais vulneráveis. Os percentuais ajustados pelo modelo bayesiano, apresentados na Figura 2, ilustram que a gravidez adolescente tende a ser menos frequente nos grandes centros urbanos, regiões estas caracterizadas por maior disponibilidade de oferta de serviços de saúde e maior renda per capita, mas onde ainda persistem a segregação, a pobreza e a desigualdade social 32 . No Brasil, enquanto estima-se que 55\% das adolescentes solteiras e sexualmente ativas não utilizam nenhum método anticoncepcional, este número eleva-se para $79 \%$ quando consideradas as residentes nas áreas rurais ${ }^{33}$. Em um estudo norte-americano ${ }^{34}$, igualmente verificou-se que a ocorrência de gravidez dos 15 aos 19 anos de idade é maior na zona rural do que nas áreas metropolitanas, onde, de uma forma geral, há mais acesso à educação e à informação 11 . Ambos os estudos sugerem que as áreas distantes dos grandes centros tendem a oferecer dificuldades de acesso a serviços de saúde e propiciam um baixo conhecimento sobre métodos anticoncepcionais. Esses dados reforçam a importância de se conhecer e considerar as especificidades do contexto para adequar práticas assistenciais no campo da saúde.

Por se tratar de um estudo de modelo ecológico, as características aqui identificadas dizem respeito a um grande contingente populacional, refletindo o fenômeno social da gravidez na adolescência em sua ocorrência coletiva, e não individual. Esse fator diferencia o presente estudo da maioria dos demais trabalhos publicados na literatura, nos quais as associações são estudadas em nível de indivíduos e a população alvo é composta por mulheres residentes em regiões menores. O desenho aqui empregado é passível do viés ecológico (ou falácia ecológica), que adverte que uma associação observada entre agregados de indivíduos não significa, necessariamente, que a mesma associação ocorra em nível de indivíduos. Entretanto, os resultados aqui encontrados possuem grande compatibilidade com aqueles dos estudos que não utilizam modelos ecológicos, e isto reforça a hipótese de que o meio em que uma adolescente vive favorece de forma marcante uma gravidez precoce: os fatores associados à chance de engravidar-se não se restringem às suas características individuais, mas alcançam as inter-relações com o ambiente em que vive.

Uma possível limitação do estudo relacionase à qualidade da informação do SINASC. Esse sistema possui boa completitude de preenchimento e consistência da informação na maioria das variáveis 35 , como a idade materna, essencial para o presente estudo, mas a possibilidade de subnotificações de registros de nascidos vivos 36 pode trazer algum viés na estimação dos percentuais de gravidez na adolescência. Desconsiderando isso, o modelo bayesiano aqui empregado mostrou-se eficiente na estimação dos percentuais ajustados, sendo que a estrutura espacial e a matriz de vizinhança adotadas mostraramse adequadas ao conjunto de dados, visto que, quando incorporadas ao modelo, os valores de DIC apresentaram grande redução. Em um contexto epidemiológico, modelos bayesianos semelhantes ao aqui empregado têm sido utilizados em outras publicações recentes 29,30, mostrando 
Diagramas de dispersão dos percentuais de gravidez na adolescência nos municípios do Estado de São Paulo e (a) IDH-M; (b) IDH-M educação;

(c) IDH-M longevidade; e (d) IDH-M renda.

a) IDH-M

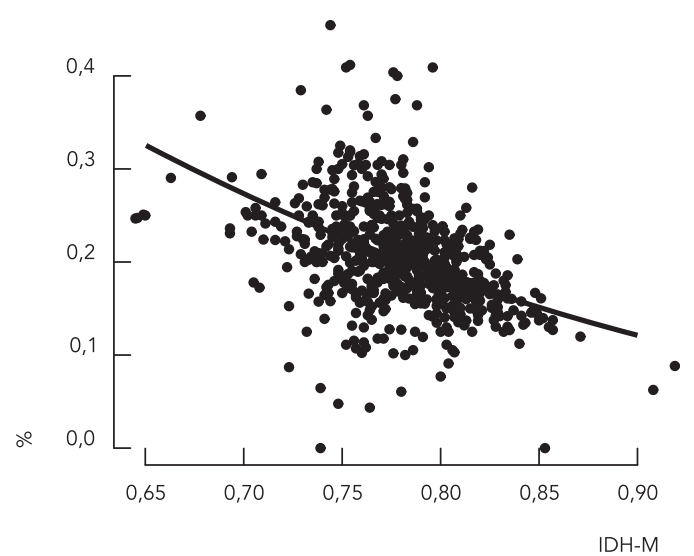

c) IDH-M longevidade

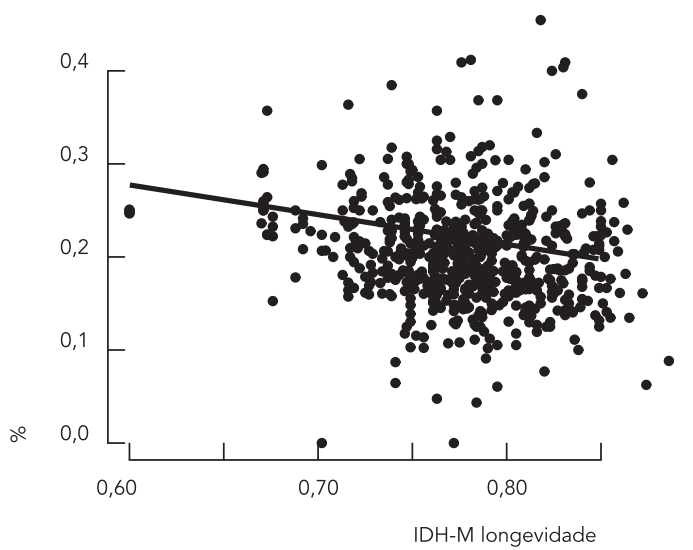

b) IDH-M educação

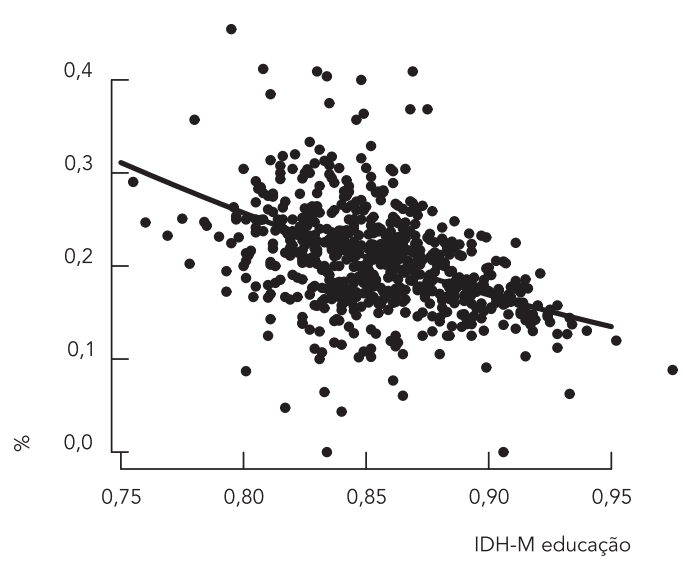

d) IDH-M renda

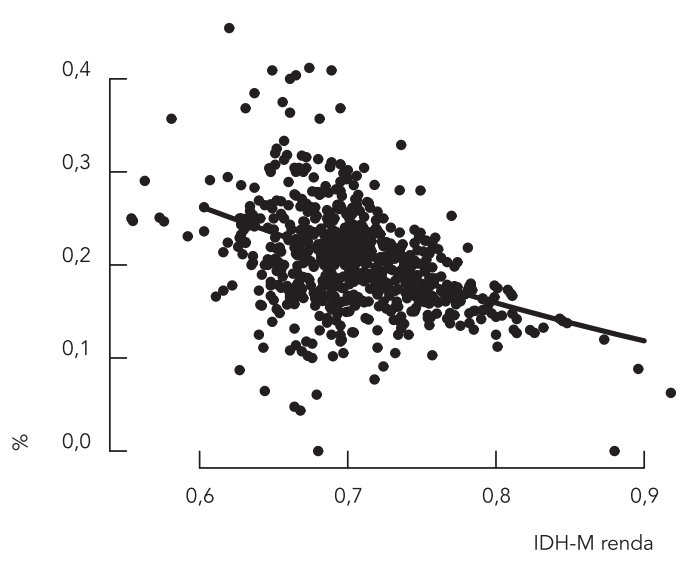

IDH-M: índice municipal de desenvolvimento humano.

Nota: as curvas exibidas nos gráficos são resultantes do ajuste dos modelos de regressão.

um bom desempenho na estimação de taxas e outras medidas de interesse.

$\mathrm{O}$ presente estudo evidenciou que os municípios com maiores valores do componente educação do IDH-M tendem a possuir menores porcentagens de gravidez na adolescência. Esse é um importante resultado, uma vez que a profunda relação entre a educação e a gravidez precoce traz como consequência a manutenção do estado de pobreza de uma comunidade, já que as adolescentes grávidas tendem a abandonar a escola 37,38 e o retorno ao estudo muitas vezes não ocorre ${ }^{39}$, com posteriores dificuldades em conseguir empregos bem remunerados. Em adição, a tendência a partos prematuros faz com que os filhos dessas adolescentes estejam expostos a atrasos no desenvolvimento neuropsicomotor e consequentes dificuldades de aprendizado escolar 40, contribuindo com novas impossibilidades de posições qualificadas no mercado de trabalho, fazendo persistir as diferenças econômicas e sociais. Na experiência clínica diária há relatos de adolescentes que optaram pela gravidez precoce, e não a evitaram com o propósito de deixar o lar 
Percentuais de gravidez na adolescência, segundo classes do índice municipal de desenvolvimento humano (IDH-M) do Estado de São Paulo, Brasil.

\begin{tabular}{|c|c|c|c|c|c|}
\hline \multirow[t]{2}{*}{ Variáveis } & \multirow[t]{2}{*}{$\begin{array}{l}\text { Número de } \\
\text { municípios }\end{array}$} & \multicolumn{2}{|c|}{$\begin{array}{l}\text { Percentual médio de gravidez na } \\
\text { adolescência }\end{array}$} & \multicolumn{2}{|c|}{ Razão entre os percentuais } \\
\hline & & $\%$ & IC95\% & Razão & IC95\% \\
\hline \multicolumn{6}{|l|}{ IDH-M (2000) } \\
\hline Até 0,728 & 33 & 24,46 & $22,67-26,37$ & \multicolumn{2}{|c|}{ Referência } \\
\hline $0,729-0,758$ & 134 & 22,75 & $21,85-23,62$ & 0,932 & $0,854-1,014$ \\
\hline $0,759-0,779$ & 157 & 21,25 & $20,59-21,91$ & 0,870 & $0,799-0,944$ * \\
\hline $0,780-0,801$ & 160 & 19,89 & $19,37-20,40$ & 0,814 & $0,748-0,883$ * \\
\hline $0,802-0,830$ & 129 & 17,35 & $16,93-17,78$ & 0,710 & $0,655-0,770$ * \\
\hline$>0,830$ & 32 & 14,62 & $13,92-15,35$ & 0,598 & $0,545-0,656$ * \\
\hline \multicolumn{6}{|l|}{ IDH-M educação } \\
\hline Até 0,805 & 33 & 23,57 & $21,68-25,34$ & \multicolumn{2}{|c|}{ Referência } \\
\hline $0,806-0,834$ & 134 & 22,91 & $22,10-23,79$ & 0,974 & $0,887-1,075$ \\
\hline $0,835-0,854$ & 160 & 21,15 & $20,60-21,72$ & 0,899 & $0,828-0,979$ * \\
\hline $0,855-0,877$ & 159 & 20,47 & $19,93-21,00$ & 0,870 & $0,798-0,953$ * \\
\hline $0,878-0,912$ & 127 & 17,57 & $17,15-17,98$ & 0,747 & $0,686-0,810$ * \\
\hline$>0,912$ & 32 & 14,17 & $13,60-14,47$ & 0,602 & $0,547-0,664$ * \\
\hline \multicolumn{6}{|c|}{ IDH-M longevidade } \\
\hline Até 0,704 & 33 & 24,04 & $22,03-26,06$ & \multicolumn{2}{|c|}{ Referência } \\
\hline $0,705-0,753$ & 134 & 20,85 & $20,05-21,67$ & 0,869 & $0,794-0,958$ * \\
\hline $0,754-0,779$ & 157 & 19,23 & $18,59-19,85$ & 0,802 & $0,733-0,876$ * \\
\hline $0,780-0,806$ & 160 & 19,05 & $18,47-19,68$ & 0,794 & $0,725-0,875$ * \\
\hline $0,807-0,847$ & 129 & 18,87 & $18,19-19,62$ & 0,787 & $0,713-0,866$ * \\
\hline$>0,847$ & 32 & 17,82 & $16,17-19,65$ & 0,743 & $0,646-0,845$ * \\
\hline \multicolumn{6}{|l|}{ IDH-M renda } \\
\hline Até 0,633 & 34 & 23,73 & $21,88-25,49$ & \multicolumn{2}{|c|}{ Referência } \\
\hline $0,634-0,671$ & 128 & 21,81 & $20,94-22,72$ & 0,921 & $0,840-1,009$ \\
\hline $0,672-0,699$ & 163 & 21,79 & $21,20-22,42$ & 0,920 & $0,845-1,002$ \\
\hline $0,700-0,734$ & 159 & 20,19 & $19,66-20,70$ & 0,852 & $0,785-0,921$ * \\
\hline $0,735-0,786$ & 129 & 17,71 & $17,33-18,12$ & 0,747 & $0,690-0,808$ * \\
\hline$>0,786$ & 32 & 14,29 & $14,31-14,95$ & 0,603 & $0,551-0,669$ * \\
\hline
\end{tabular}

IC95\%: intervalo de $95 \%$ de credibilidade.

* Diferenças significantes em relação à classe de referência.

de seus pais, devido às precárias condições de vida a que estavam submetidas. Todavia, acabam por ampliar as dificuldades de sobrevivência anteriormente sentidas.

As repercussões da gravidez na adolescência na escolaridade têm sido estudadas por diversos autores 39,41,42,43. Em um trabalho sobre as características da trajetória escolar de jovens em três capitais brasileiras ${ }^{44}$, observou-se que a metade das que interromperam os estudos pelo menos uma vez relatou uma gravidez na adolescência. Neste estudo, verificou-se que o abandono escolar na ocasião da gravidez na adolescência foi referido por $40,1 \%$ das moças cuja gravidez ter- minou em nascimento de um filho. Em um estudo sobre indicadores sociais em uma população de gestantes 45 , estimou-se que apenas $6,8 \%$ das adolescentes eram estudantes. Entretanto, se por um lado a gravidez na adolescência pode ocasionar a evasão escolar, muitas adolescentes podem ter deixado a escola antes de engravidar, sendo por sua vez esta evasão um possível fator de risco para a gravidez precoce ${ }^{38}$. Em adição, filhos adolescentes de mulheres com um ano de escolaridade têm probabilidade 23 vezes maior de chegar analfabeto à adolescência, em comparação com filhos de mulheres com 11 anos ou mais de escolaridade 46 . 
As associações entre o IDH-M e os percentuais de gravidez na adolescência são também apontadas em um estudo sobre a fecundidade e os diferenciais intraurbanos de desenvolvimento humano, conduzido no Município de São Paulo 47. Este estudo indicou que, ao considerarse o município como um todo, as adolescentes responderam por $30,9 \%$ dos nascimentos dentre as primíparas, mas este percentual reduziu-se para $20,2 \%$ quando considerada a área de maior IDH.

Sendo a gravidez adolescente contemplada como um fenômeno relacionado a um contexto de vulnerabilidade social, é necessário considerar que os resultados do presente estudo evidenciam que sua frequência ocorre em maior intensidade em ambientes pontuados por oportunidades restritas e poucas opções de vida. Isso pode ser concluído com base na observação de que municípios com menores IDH e com maiores incidências de pobreza são aqueles que apresentam maiores percentuais de gravidez adolescente. Além disso, esses percentuais associam-se a baixos níveis de escolaridade, lembrando que a literatura aponta que as interrupções na trajetória escolar que antecedem a gravidez perpetuam uma situação de exclusão social por várias gerações de mulheres de uma mesma família 43 . Municípios desfavorecidos em termos de desen- volvimento humano trazem um contexto de desvantagens sociais para a adolescente, que supostamente coloca a maternidade como uma alternativa de vida, dentre poucas oportunidades que o ambiente favorece. Essas inter-relações entre as adolescentes e o contexto social propiciado pelo ambiente em que vivem são de grande importância para o conhecimento dos fatores que levam à gestação. Incentiva-se que essas inter-relações sejam melhor exploradas em novos estudos de natureza ecológica.

As intervenções diretas sobre grupos vulneráveis de adolescentes, como campanhas de prevenção e ações educativas, são de grande importância para a prevenção de gravidezes precoces, mas os achados do presente estudo sugerem que o problema tem dimensões mais amplas, e resultados mais eficientes seriam obtidos a partir de ações que incrementem ou otimizem os recursos dos municípios para que compensem diferenças sociais prévias. Dessa forma, seu enfrentamento transcende o setor saúde, requerendo práticas e saberes intersetoriais e a possibilidade de estabelecimento de linhas de cuidado que incluam aspectos que abrangem desde as relações intersubjetivas e da microgestão do cuidado, até aspectos da macrogestão da saúde, que no momento fogem do âmbito e dos objetivos do presente estudo, mas cuja necessidade fica colocada.

\section{Resumo}

A gravidez na adolescência é um problema de saúde pública comum em todo o mundo. O objetivo deste estudo ecológico é estudar o padrão espacial da associação entre os percentuais de gravidez na adolescência e características socioeconômicas dos municípios do Estado de São Paulo, Brasil. Para isso, foi utilizado um modelo bayesiano com uma distribuição espacial que segue uma estrutura condicional autorregressiva (CAR), baseado em algoritmos Monte Carlo em cadeias de Markov (MCMC). Foram usados dados do Sistema de Informações sobre Nascidos Vivos (SINASC) e do Instituto Brasileiro de Geografia e Estatística (IBGE). Verificou-se que a ocorrência de gravidezes precoces apresentou-se maior nos municípios de menor produto interno bruto (PIB) per capita, com maior incidência de pobreza, de menor tamanho populacional, menor índice de desenvolvimento humano (IDH) $e$ maior percentual de indivíduos com índice paulista de vulnerabilidade social (IPVS) igual a 5 ou 6, ou seja, mais vulneráveis. O estudo demonstra uma estreita associação entre gravidez na adolescência e indicadores econômicos e sociais.

Gravidez na Adolescência; Indicadores Básicos de Saúde; Análise Espacial 


\section{Colaboradores}

E. Z. Martinez participou da concepção do projeto, montagem do banco de dados, análise e interpretação dos dados. D. L. Roza contribuiu no desenho do estudo, análise estatística, interpretação e discussão dos dados. M. C. G. G. Caccia-Bava e A. L. Dal-Fabbro contribuíram na análise e interpretação dos dados. J. A. Achcar colaborou na análise estatística, interpretação e discussão dos dados. Todos os autores participaram da redação do manuscrito e aprovaram a sua versão final.

\section{Referências}

1. Outeiral JO. Adolescer: estudo sobre adolescência. Porto Alegre: Editora Artes Médicas; 1994.

2. Cano MAT, Ferriani MGC, Medeiros M, Gomes R. Auto-imagem na adolescência. Rev Eletr Enf 1999; 1(1). http://www.fen.ufg.br/revista/revista1_1/Au to.html.

3. Cunha AA, Monteiro DLM. Gravidez na adolescência como problema de saúde pública. In: Monteiro DLM, Cunha AA, Bastos AC, organizadores. Gravidez na adolescência. Rio de Janeiro: Revinter; 1998; p. 31-42.

4. Heilborn ML, Salem T, Rohden F, Brandão E, Knauth D, Victora C, et al. Aproximações socioantropológicas sobre a gravidez na adolescência. Horizontes Antropológicos 2002; 8:13-45.

5. Oliveira MW. Gravidez na adolescência: dimensões do problema. Cad CEDES 1998; 19:48-70.

6. Gama SGN, Szwarcwald CL, Leal MC. Experiência de gravidez na adolescência, fatores associados e resultados perinatais entre puérperas de baixa renda. Cad Saúde Pública 2002; 18:153-61.

7. Costa MCO, Santos CAST, Nascimento Sobrinho C, Moura MSQ, Souza KEP, Assis DR. Gravidez na adolescência: associação de variáveis sociodemográficas e biomédicas maternas com resultado neonatal. Feira de Santana - BA. Rev Baiana Saúde Pública 2005; 29:300-12.

8. Goldenberg P, Figueiredo MC, Silva RS. Gravidez na adolescência, pré-natal e resultados perinatais em Montes Claros, Minas Gerais, Brasil. Cad Saúde Pública 2005; 21:1077-86.

\section{Agradecimetos}

Os autores agradecem à Fundação de Apoio ao Ensino, Pesquisa e Assistência (FAEPA) do Hospital das Clínicas da Faculdade de Medicina de Ribeirão Preto da Universidade de São Paulo, pelo apoio financeiro.
9. Halpern R, Giugliani ERJ, Victora CG, Barros FC, Horta BL. Fatores de risco para suspeita de atraso no desenvolvimento neuropsicomotor aos $12 \mathrm{me}-$ ses de vida. Rev Chil Pediatr 2002; 73:529-39.

10. Sociedade Civil Bem-Estar Familiar no Brasil. Adolescentes, jovens e a Pesquisa Nacional sobre Demografia e Saúde: um estudo sobre fecundidade, comportamento sexual e saúde reprodutiva. Rio de Janeiro: Sociedade Civil Bem-Estar Familiar no Brasil; 1999.

11. Chalem E, Mitsuhiro SS, Ferri CP, Barros MC, Guinsburg R, Laranjeira R. Gravidez na adolescência: perfil sociodemográfico e comportamental de uma população da periferia de São Paulo, Brasil. Cad Saúde Pública 2007; 23:177-86.

12. Duarte CM, Nascimento VB, Akerman M. Gravidez na adolescência e exclusão social: análises de disparidades intra-urbanas. Rev Panam Salud Pública 2006; 19:236-43.

13. Carniel EF, Zanolli ML, Almeida CAA, Morcillo AM. Características das mães adolescentes e de seus recém-nascidos e fatores de risco para a gravidez na adolescência em Campinas, SP, Brasil. Rev Bras Saúde Matern Infant 2006; 6:419-26.

14. Amorim MMR, Lima LA, Lopes CV, Araújo DKL, Silva JGG, César LC, et al. Fatores de risco para a gravidez na adolescência em uma maternidadeescola da Paraíba: estudo caso-controle. Rev Bras Ginecol Obstet 2009; 31:404-10. 
15. Boruchovitch E. Fatores associados a não-utilização de anticoncepcionais na adolescência. Rev Saúde Pública 1992; 26:437-43.

16. Guimarães AMAN, Vieira MJ, Palmeira JA. Informações dos adolescentes sobre métodos anticoncepcionais. Rev Latinoam Enferm 2003; 11:293-8.

17. Caputo VG, Bordin IA. Gravidez na adolescência e uso freqüente de álcool e drogas no contexto familiar. Rev Saúde Pública 2008; 42:402-10.

18. Nogueira MJ, Silva BFA, Barcelos SM, Schall VT. Análise da distribuição espacial da gravidez adolescente no Município de Belo Horizonte - MG. Rev Bras Epidemiol 2009; 12:297-312.

19. Gastwirth JL. The estimation of the Lorenz curve and Gini index. Rev Econ Stat 1972; 54:306-16.

20. Jannuzzi PM. Indicadores para diagnóstico, monitoramento e avaliação de programas sociais no Brasil. Revista do Serviço Público 2005; 56:137-60.

21. Jorge MHPM, Laurenti R, Gotlieb SLD. Análise da qualidade das estatísticas vitais brasileiras: a experiência de implantação do SIM e do SINASC. Ciênc Saúde Coletiva 2007; 12:643-54.

22. Fundação Sistema Estadual de Análise de Dados. Índice paulista de vulnerabilidade social - IPVS: espaços e dimensões da pobreza nos municípios do Estado de São Paulo. http://www.seade.gov.br/ produtos/ipvs/pdf/oipvs.pdf (acessado em 10/ $\mathrm{Jul} / 2010)$.

23. Ferreira MP, Dini NP, Ferreira SP. Espaços e dimensões da pobreza nos Municípios do Estado de São Paulo: índice paulista de vulnerabilidade social IPVS. São Paulo Perspect 2006; 20:5-17.

24. Ashby D. Bayesian statistics in medicine: a 25 year review. Stat Med 2006; 25:3589-631.

25. Carvalho MS, Souza-Santos R. Análise de dados espaciais em saúde pública: métodos, problemas, perspectivas. Cad Saúde Pública 2005; 21:361-78.

26. Besag J, Kooperberg CL. On conditional and intrinsic autoregressions. Biometrika 1995; 82:733-46.

27. Thomas A, Best N, Lunn D, Arnold R, Spiegelhalter D. GeoBUGS user manual. Cambridge: Medical Research Council Biostatistics Unit; 2004.

28. Lunn DJ, Thomas A, Best N, Spiegelhalter D. WinBUGS - a Bayesian modelling framework: concepts, structure, and extensibility. Stat Comput 2000; 10:325-37.

29. Assunção RM, Barreto SM, Guerra HL, Sakurai E. Mapas de taxas epidemiológicas: uma abordagem Bayesiana. Cad Saúde Pública 1998; 14:713-23.

30. Bernardinelli L, Clayton D, Pascutto C, Montomoli C, Ghislandi M, Songini M. Bayesian analysis of space-time variation in disease risk. Stat Med 1995; 14:2433-43

31. Spiegelhalter DJ, Best NG, Carlin BP, van der Linde A. Bayesian measures of model complexity and fit (with discussion). J R Stat Soc Series B Stat Methodol 2002; 64:583-639.

32. Viana ALA, Rocha JSY, Elias PE, Ibañez N, Novaes MHD. Modelos de atenção básica nos grandes municípios paulistas: efetividade, eficácia, sustentabilidade e governabilidade. Ciênc Saúde Coletiva 2006; 11:577-606.

33. Bennett T, Skatrud JD, Guild P, Loda F, Klerman LV. Rural adolescent pregnancy: a view from the South. Fam Plann Perspect 1997; 29:256-60.
34. Secretaria Executiva de Coordenação da Saúde da Criança e Adolescente. Programa de Saúde do Adolescente: bases programáticas. 2a Ed. Brasília: Ministério da Saúde; 1996.

35. Romero DE, Cunha CB. Avaliação da qualidade das variáveis epidemiológicas e demográficas do Sistema de Informações sobre Nascidos Vivos, 2002. Cad Saúde Pública 2007; 23:701-14.

36. Drumond EF, Machado CJ, França E. Subnotificação de nascidos vivos: procedimentos de mensuração a partir do Sistema de Informação Hospitalar. Rev Saúde Pública 2008; 42:55-63.

37. Moreira TMM, Viana DS, Queiroz MVO, Bessa Jorge MS. Conflitos vivenciados pelas adolescentes com a descoberta da gravidez. Rev Esc Enferm USP 2008; 42:312-20.

38. Yazlle MEHD, Mendes MC, Patta MC, Rocha JSY, Azevedo GD, Marcolin AC. A adolescente grávida: alguns indicadores sociais. Rev Bras Ginecol Obstet 2002; 24:609-14.

39. Molina M, Ferrada C, Perez R, Cid L, Casanueva V, Garcia A. Embarazo en la adolescencia y su relación con la deserción escolar. Rev Méd Chil 2004; 132:65-70.

40. Méio MD, Lopes CS, Morsch DS, Monteiro APG. Desenvolvimento cognitivo de crianças prematuras de muito baixo peso na idade pré-escolar. J Pediatr (Rio J.) 2004; 80:495-502.

41. Barnet B, Arroyo C, Devoe M, Duggan AK. Reduced school dropout rates among adolescent mothers receiving school-based prenatal care. Arch Pediatr Adolesc Med 2004; 158:262-8.

42. Grant MJ, Hallman KK. Pregnancy-related school dropout and prior school performance in KwaZulu-Natal, South Africa. Stud Fam Plann 2008; 39:369-82.

43. Almeida MCC, Aquino EML. The role of education level in the intergenerational pattern of adolescent pregnancy in Brazil. Int Perspect Sex Reprod Health 2009; 35:139-46.

44. Almeida MCC, Aquino EML, Barros AP. School trajectory and teenage pregnancy in three Brazilian state capitals. Cad Saúde Pública 2006; 22:1397409.

45. Michelazzo D, Yazlle MEHD, Mendes MC, Patta MC, Rocha JSY, Moura MD. Indicadores sociais de grávidas adolescentes: estudo caso-controle. Rev Bras Ginecol Obstet 2004; 26:633-9.

46. Buss PM, Pellegrini Filho A. Iniqüidades em saúde no Brasil, nossa mais grave doença: comentários sobre o documento de referência e os trabalhos da Comissão Nacional sobre Determinantes Sociais da Saúde. Cad Saúde Pública 2006; 22:2005-8.

47. Martins CM, Almeida MF. Fecundidade e diferenciais intra-urbanos de desenvolvimento humano, São Paulo, Brasil, 1997. Rev Saúde Pública 2001; 35:421-7.

Recebido em 14/Jul/2010

Versão final reapresentada em 16/Fev/2011

Aprovado em 24/Fev/2011 